\title{
মুসলিম ঐতিহ্য ও নজরুল সাহিত্য
}

\section{Muslim Traditions and the Literary Works of Nazrul}

\author{
ড. রিটা আশরাফ*
}

সার সংক্ষেপ: উপমহাদেশে উপনিবেশবাদের এক চরম সংকটময় অবস্থার মাঝে কাজী নজরুল ইসলামের আবির্ভাব ঘটে বাংলা সাহিত্যে। কাজী নজরুল ইসলাম বিশ ও ত্রিশের দশকে উপমহাদেশের অবিভক্ত বাংলার সাহিত্যাঙ্গনে এক ব্যতিক্রমধর্মী ব্যক্তিত্ব ছিলেন। সাহিত্য রচনার নানা মুখিতায় মুসলমানদের ধর্মীয় ঐতিহ্যও নজরুল রচনার একটি অন্যতম অধ্যায়। মুসলিম ঐতিহ্যের নানা দিক নিয়ে নজরুল লিখেছেন। নজরুল তাঁর কবিতা, গান ও সাহিত্যের অন্যান্য শাখার ভেতর মুসলমানদের ইতিহাস ও ঐতিহ্য তুলে ধরেছেন। হযরত মুহাম্মদ সা.-এর জীবনী, মুসলমানদের পবিত্র ধর্মগ্রন্থ কুরআন শরীফসহ ইসলামের নানা ইতিহাস নিয়ে লিখেছেন। প্রায় দুই সহস্রাধিক গান রয়েছে নজরুলের। যেখানে স্থান পেয়েছে ইসলাম ধর্মের আরো নানা বিষয়। যেমন- নামাজ, রোজা, ঈদ, হজ্ব, জাকাত, হযরত মুহাম্মদ সা.-এর মাতা, পিতা, স্ত্রীসহ বিভিন্ন নবি, রাসুল এবং মুসলিম জাহানের উল্লেখযোগ্য ব্যক্তিবর্গ। কাব্য আমপারার মধ্যে দিয়ে পবিত্র কুরআন শরীফের বঙ্গানুবাদ করতে চেয়েছিলেন নজরুল। হযরত মুহাম্মদ সা.-এর জীবনী প্রকাশ করতে চেয়েছিলেন মরুভাস্কর কাব্যের মধ্যে দিয়ে। "মুসলিম ঐতিহ্য ও নজরুল সাহিত্য" শীর্ষক আলোচ্য প্রবন্ধটিতে এই বিষয়গুলো আলোচনা-পর্যালোচনা করার প্রয়াস রয়েছে।

মূল শব্দসমূহ : সাহিত্য, নজরুল, মুসলিম ঐতিহ্য, ধর্ম এবং ইসলাম।

Abstract: Kazi Nazrul Islam came in the field of Bengali literature at a crucial stage of colonialism in the Indian sub-continent. He was a literary personality of the undivided Bengal with different characteristics. One of the major focuses of his literary works is Muslim religious traditions with various aspects of Islamic values. He highlighted Muslim history and traditions in his poems, songs and other literary forms. He wrote about the life of Prophet Muhammad (SAAS), the Holy Quran-the religious book of the Muslims and other historical elements of Islam. He composed more than two thousand songs dealing with various Islamic subjects such as the Prayer, the Fasting, the Hajj, the Zakat; the parents and wives of Hazrat Muhammad (SAAS); other Prophets and Messengers; as well as the renowned personalities of the Muslim world. He started to translate the Holy Quran, and 'Kabbya Ampara' is the outcome of his noble initiation in this regard. His poetry the 'Maru

\footnotetext{
* ড. রিটা আশরাফ: সহয়াগী অধ্যাপক ও বিভাগীয় প্রধান, বাংলা বিভাগ, এশিয়ান ইউনিভার্সিটি অব বাংলাদেশ। E-mail:
} ritaashraf2009@gmail.com 
Bhaskar' is a biographical sketch of Prophet Muhammad (SAAS). All these aspects of Nazrul's literary works have been discussed and critically analyzed in this article under the title 'Muslim Traditions and the Literary Works of Nazrul'.

Keywords: Literature, Nazrul, Muslim traditions, religion and Islam

ভূমिका

$$
\begin{aligned}
& \text { “ইসলাম বলে, সকলের তরে মোরা সবাই } \\
& \text { সুখ-দুখ সম-ভাগ করে নেব সকলে ভাই, } \\
& \text { নাই অধিকার সঞ্চয়ের!” }
\end{aligned}
$$

নজরুল সাহিত্যে মুসলিম ঐতিহ্য একটি গুরুত্বপূর্ণ অধ্যায়। ‘ঈদ-মোবারক’ কবিতার উক্ত উদ্ধৃতিটি “মুসলিম ঐতিহ্য ও নজরুল সাহিত্য” শীর্ষক আলোচ্য প্রবন্ধটিতে বিশেষভাবে প্রভাব বিস্তার করে আছে। নজরুল ইসলাম তাঁর কবিতা, গান, গল্প, নাটক, প্রবন্ধ, রাজনৈতিক কর্মকাণ্ ইত্যাদির মধ্য দিয়ে তৎকালীন অর্থাৎ নজরুল সমকালীন পরাধীন ভারতে বিশেষ করে অবিভক্ত বাংলাদেশে পরাধীনতা, সাম্পদ্রদয়িকতা, মৌলবাদ, সামন্তবাদ, সাম্রাজ্যবাদ, উপনিবেশবাদ এবং দেশি-বিদেশি শাসন ও শোষণের বিরুদ্ধে বিদ্রোহের ঝাল্ডা উড়িয়েছিলেন। প্রথম বিশ্বযুদ্ধোত্তর ভারতবর্ষের রাজনৈতিক আর্থ-সামাজিক এবং সমকালীন নানা ঘটনাকে সাহিত্যের পটভূমি করে তুলেছিলেন। এজন্য নজরুল ইসলামকে ইংরেজ সরকারের কাছে জবাবদিহির কাঠগড়ায়ও দাঁড়াতে হয়েছিল। ইংরেজ সরকার নজরুলের একের পর এক গ্রন্থ নিষিদ্ধ করেই ক্ষান্ত হয়নি, নজরুলকে কারাদণ্ণ দণ্ডিত করেছিল। এ থেকেই বুঝা যায়, উপনিবেশ শাসন নজরুলকে যথেষ্টই ভয় করে চলত। বাংলা সাহিত্যের প্রতিটি শাখায় নজরুল বিচরণ করেছেন একান্তই নজরুলীয় রীতিতে। এমনকি অনুবাদ সাহিত্যেও যথেষ্ট পারদর্শিতার এবং নিপূণতার সাক্ষর রেখে গেছেন নজরুল। নজরুল যখন বাংলা সাহিত্যে “একহাতে বাঁতের বাঁশরি, আর হাতে রণতূর্য” নিয়ে আবির্ভূত হলেন তখন বাংলা সাহিত্যের একাধিপতি সম্রাট ছিলেন রবীন্দ্রনাথ ঠাকুর। এ সময়কালের প্রায় সব লেখকগণই তখন রবীন্দ্র জয়গানে লীন। রবীন্দ্র বলয় থেকে বের হয়ে আসার সেই মনোবল বা লেখার ঐশ্বর্যও কারো ছিল না। ঠিক তখনই নজরুল এলেন ভিন্ন এক স্বর নিয়ে। রবীন্দ্র বলয় থেকে সবার দৃষ্টি ঘোরালেন এক নতুন স্রষ্টার দিকে। যার অগ্নিবীণার সুরে মোহভঙ্গ হল সবার। আসন দুলে উঠল উপনিবেশবাদ শাসনের। ১b-৯৯ সালে জনুগ্রহণ করে জনেমের দশ বছরের মধ্যেই নজরুল নিজের দিকে নিজের অজান্তেই মানুষের চোখ ফেরাতে সক্ষম হন তাঁর সৃষ্টিশীল ও সৃজনশীল অতুলনীয় কর্র্মের মাধ্যন্।ে। সাহিত্য জগতে এক অলৌকিক সৃষ্টিশীল ক্ষমতা নিয়ে এগুতে ওুরু করেন নজরুল। নজরুলের ইসলামি চেতনামূলক রচনাও এ সময় বিশেষ ভূমিকা পালন করে।

নজরুলের সমগ্র রচনা ঢাঁর মৌলিক চেতনায় সমৃদ্ধ। নজরুল যেমন লিখেছেন উপনিবেশিক শাসন শোষণের বিরুদ্ধে, সাম্প্রদায়িকতার বিরতদ্ধে, তেমনি ধর্মীয় চেতনামূলক রচনাও রয়েছে তাঁর প্রচুর। হিন্দু বৌদ্ধ, খ্রিষ্টান, মুসলমান সকল ধর্মের প্রবাহ নজরুল সাহিত্যে রয়েছে। নজরুল সাহিত্যের একটি বিরাট অংশ জুড়ে রয়েছে 
ইসলাম ধর্ম ও মুসলমান সম্প্রদায়। তিনি কখনো ইসলাম ধর্মকে পুঁজি করে ফতোয়াবাজ, পীর ফকিরদের রমরমা ব্যবসার মুখোশ উন্মোচন করেছেন, কখনো ইসলামি ঐতিহ্যের প্রবাহে পাঠক হৃদয় ভাসিয়ে দিয়েছেন। এক্ষেত্রে কবিতা ও গানেই তিনি বেশি বিচরণ করেছেন।

\section{নজরুল রচনায় মুসলিম ঐতিহ্য}

ফাতেহা-ই দোয়াজদহম কবিতটি ১৩২৭ সালের মোসলেম ভারত অগ্রহায়ণ সংখ্যায় প্রকাশিত হয়। আবির্ভাব ও তিরোভাব নান্ দু’টি অংশে সংকলিত কবিতাটি ইসলাম ধর্মের প্রবর্তক হযরত মুহাম্মদ সা.-কে নিয়ে রচিত। হযরত মুহাম্মদ সা.-এর পৃথিবীতে আগমন উপলক্ষ্যে রচিত হয় আবির্ভাব। “মহানবি সা.-এর জন্ম মুহূর্তেকে দুনিয়া ও বেহেশতের শুভ ও আনন্দময় লগুরূপে উল্লেখ করার পাশাপাশি মুসলমানদের অতীত ইতিহাস, ঐতিহ্য, মুসলিম বিশ্বের ভূগোল ইত্যাদিরও উল্লেখ রয়েছে কবিতাটিতে। সাথে সাথে মুসলিম জাহানের গুরতত্বপূর্ণ স্থানেরও নাম উল্লেখ রয়েছে কবিতাটিতে। ভৌগলিক ও ঐতিহাসিক চেতনাকে জাগিয়ে তোলার জন্য কবিতায় নজরুল বলেছেন:

নाই তা-জ

তাই লা-জ?

ওরে মুসলিম, খর্জুর শীযে তোরা সাজ!

করে তসলিম, হর কুর্ণিশে শোর্-আওয়াজ

শোন্ কোন মুরদা সে উচ্চারে ‘হেরা’ আজ

ধরা মাঝ!

এখানে সমকালীন মুসলমানদের হীনমন্যতা দূর করে আপন গৌরবে তাদের সাজতে বলা হয়েছে। হযরত মুহাম্মদ সা.-এর সমকালীন ভৌগলিক ও ঐতিহাসিক চেতনাকে আনন্দের সাথে, উল্লাসের সাথে প্রকাশ করা হয়েছে। প্রকাশ করা হয়েছে হযরত মুহাম্মদ সা. হেরা পর্বতের গুহায় আধ্যাত্মিক চেতনা লাভ করার ফনেই ইসলাম ধর্ম প্রচার হয়েছিল।

ফাতেহা-ই-দোয়াজদহম (তিরোভাব) কবিতাটিতে মহানবি সা.-এর তিরোধান বিষয়টি পরিস্ফুট হয়েছে।

আজ স্বরগের হাসি ধরার অশ্রু ছাপায়ে অবিশ্রাম

ওঠে একী ঘন রোল- সাল্লালাহো আলায় হিসাল্লাম।

নবি দুহিতা ফাতেমা রা.’র কান্না, হাসান হোসেন রা.-এর আহাজারির বর্ণনা, মুয়াজ্জিনের কম্পিত কন্ঠে আযান ইত্যাদি চিত্র কবিতাটিতে রয়েছে।

আবির্ভাব প্রথম প্রকাশিত হয় মোসলেম ভারত, ১৩২৭ অগ্রহায়ণ সংখ্যায় এবং তিরোভাব প্রকাশিত হয় মোসলেম ভারত ১৩২৮- সালের অগ্রহায়ণ সংখ্যায়

নজরুলের কোরবানী’ কবিতা সরাসরি ইসলাম ধর্ম্মের নানা আচার অনুষ্ঠানের বিষয়ভিত্তিক একটি কবিতা। কবিতাটি মুসলমানদের একটি অন্যতম ধর্মীয় অনুষ্ঠান কুরবানীর ঈদ নিয়ে রচিত। কবিতাটিতে কবি ইসলাম ধর্ম্মের ঈদুল আজহার তাৎপর্যকে এভাবে তুলে ধরেছেন: 
ওরে হত্যা নয় আজ ‘সত্য-গ্রহ’ শক্তির উদ্বোধন!

এই দিনই ‘মীনা'-ময়দানে

পুত্র-স্নেহের গর্দানে

ছুরি হেনে’ খুন ক্ষরিয়ে নে’

রেখেছে আবববা ইবৃরাহীম সে আপনা রুদ্র পণ!

কবিতাটিতে নজরুল কুরবাণির তাৎপর্যকেই স্পষ্ট করেছেন। সাথে সাথে ইসলানের একটি ইতিহাসকে তুলে ধরেছেন। কবিতাটিতে একদিকে যেমন হয়রত ইবরাহিম আ.-এর পুত্র ইসমাইল আ.-কে কুরবানি দেয়ার মহান ত্যাগের কথা উল্লেখ আছে তেমনি উল্লেখ আছে কুরবাণির নাহে একশ্রেণির লোকের মিথ্যা অহমিকার কথা। যেমন:

চাহিনাক’ গাভী দুম্বা উট,

কতটুকু দান? ও দান ঝুট।

চাই কোরবানী, চাই না দান।

রাখিতে ইজ্জত ইসলামের

শির চাই তোর, তোর ছেলের

দেবে কি? কে আছ মুসলামান?

কুরবাণিকে নিয়ে নজরুল লিখেছেন শহীদী ঈদ, বকরীদ কবিতাসহ আরো কয়েকটি কবিতা ও গান।

কারবালার প্রান্তরে হযরত ইমাম হোসেনের শাহাদত বরণের করুণ পরিণতির ঘটনার বর্ণনা রয়েছে ‘মোহররম’ কবিতাটিতে:

বেজেছে নাকাড়া, হঁঁকে নকীবের তুর্য,

হুশিয়ার ইসলাম, ডুবে তব সূর্য?

জাগো, ওঠো মুসলিম, হাঁকো হায়দরী হাঁক,

শহীদের দিলে সব লালে লাল হয়ে যাক।

প্রায় দুই হাজারেরও বেশি ইসলামি ও মুসলিম ঐতিহ্য নির্ভর গান নজরুল লিখেছেন। যা বাংলা সংগীতেরও বিশেষ অলংকার। আমাদের রমজানের ঈদ নজরুলের যে গানটি ছাড়া পূর্ণ হয় না:

ও মন রমজানের ঐ রোজার শেষে এল খুশীর ঈদ।

তুই আপনাকে আজ বিলিয়ে দে শোন্ আসমানী তাগিদ।।

তোর সোনাদানা বালাখানা সব রহে লিল্লাহ্.

দে যাকাত্, মুর্দা মুসলিমের আজ ভাঙাইতে নিদ।।

তুই পড়বি ঈদের নামাজ রে মন সেই সে ঈদগাহে

যে ময়দানে সব গাজী মুসলিম হয়েছে শহীদ্।। 
এমন অনেক গান আজ মুসলমান সম্প্রদায়ের নানা ধর্মীয় অনুষ্ঠানের সাথে একাত্ন হয়ে আছে। যে গান ছাড়া আমাদের ধর্মীয় জীবন প্রবাহ ও নানা ধর্মীয় অনুষ্ঠান সম্পূর্ণ হয় না:

১। ত্রিভূবনের প্রিয় মুহাম্মদ এল রে দুনিয়ার।

আয়রে সাগর আকাশ বাতাস, দেখবি যদি আয়।।

ধূলির ধরা বেহেশৃতে আজ

জয় করিল, দিলরে লাজ

আজকে খুশীর ঢল নেন্মেছে

ধূসর সাহারায়

দেখ আমিনা মায়ের কোলে

দোলে শিশু ইসলাম দোলে

কচি মুখে শাহাদতের

বাণী সে শোনায়।।

২। ইসলামের ঐ বাগিচাতে ফুটলো দুটি ফুল।

শোভায় অতুল সে ফুল

আমার আল্লা ও রসুল।।

যুগল কুসুম উজল রঙে

হৃদয় আমার উঠলো রেঙে

খোশবুতে তার মাতোয়ারা মনের বুলবুল।।

৩। নামাজ পড় রোজা রাখ, কলমা পড় ভাই।

তোর আখেরের কাজ করে নে, সময় যে আর নাই।।

সম্বল যার আছে হাতে। হজের তরে যা “কাবা” তে

যাকাত দিয়ে বিনিময়ে শাফায়াত যে পাই।।

৪। ওরে ও দরিয়ার মাঝি! মোরে নিয়ে যা রে মদিনা।

তুমি মুর্শিদ হয়ে পথ দেখাও ভাই আমি যে পথ চিনি না।।

৫। দে যাকাত, দে যাকাত, তোরা দে রে যাকাত।

তোর দিল খুলবে পরে- ওরে আগে খুলুক হাত।।

৬। তৌহিদের মুর্শিদ আমার মোহাম্মদের নাম, মুর্শিদ মোহাম্মদের নাম!

ঐ নাম জপিলেই বুঝতে পারি খোদা কালাম, মুর্শিদ মোহাম্মদের নাম।। ১৯ 
१। ইসলামের ঐ সওদা লয়ে এল নবীন সওদাগর।

বদনসীব আয়, আয় গুনাহগার, নতুন করে সওদা কর।।

‘খেয়া-পারের তরণী’ কবিতাটিতে খোলাফায়ে রাশেদীনের চার খলিফার উল্লেখের ভেতর দিয়ে মুসলমানদের শেষ বিচারের দিনের তাৎপর্য ব্যাখ্যা করেছেন নজরুল এবং সেই বিচারের দিনে জয়ী হতে হনে কি করতে হবে তারও দিক নির্দেশনা দিয়েছেন এভাবে:

আবুবকর উস্মান উমর আলী হায়দর

দাড়ি যে এ তরণীর, নাই ওরে নাই ডর!

কাণ্গরী এ তরীর পাকা মাঝি মাল্লা,

দাড়ি-মুখে সারি গান-লা শরীক আল্লাহ!

কবিতাটির বিষয়বস্তু সম্পূর্ণরূপে ধর্মীয়। ধর্মীয় একটি বিষয়কে নজরুল তার সাহিত্যে এমনভাবে উপস্থাপন করেছেন যে কবিতাটি পাঠের পর যেকোনো মুসলমান হৃদয় কেঁদে উঠে। জান্নাতের পথ অনুসারী হয়ে চলতে অনুপ্রাণিত হয়। কবিতাটির মৌলিকতা এবং নির্মাণ কৌশল এখানে গুরুত্বপূর্ণ ভূমিকা রেখেছে।

‘রণভেরী’ কবিতাটিতেও ইসলামিক অনুভূতি বিরাজমান। “ওরে আয়, ঐ ইসলাম ডুবে যায়।” কবিতাটির এই একটি মাত্র পংক্তির মধ্যে দিয়েই সমগ্র মুসলমান জাতি জেগে ওঠে।

বলা যায় কাজী নজরুল ইসলাম ধ্যানে, জ্ঞানে খাঁটি মুসলমান ছিলেন। আর তাই ইসলান্মের ক্রান্তিকাল কাটিয়ে মুসলিম সমাজকে নানাভাবে জাগিয়ে তোলার চেষ্টা করেছেন তার লেখনির মধ্য দিয়ে। এ প্রসঙ্গে একটি উদ্ধৃতি:

বিরামহীন কাজের মাঝে মাঝে মুসলিম সমাজকে জাগানো, তাদের অতীত গৌরব ফিরিয়ে আনার ব্যাপারে সক্রিয় করে তোলা, আচার-অনুষ্ঠান ও কুসংস্কার সর্বস্বতার পঙ্কে নিমজ্জিত এ সমাজকে ইসলানের মহান মানবিক মূল্যবোধে উত্তীর্ণ করে সংগ্রামী জীবন তৃষ্ণায় উদ্দীপ্ত করা এ স্বপ্ন দেখা ও কর্মধারায় কখনো তাঁর (নজরুলের) বিরতি ছিল না ১৩

কামাল পাশা, আনোয়ার, চিরঞ্জীব জগলুল, আমানুল্লাহ, জামাল উদ্দীন, রীফ সর্দার, উমর ফারুক, খালেদ ইত্যাদি কবিতায় কোথাও সরাসরি ইসলামি ইতিহাস, কোথাও খ্যাতিমান মুসলমান ব্যক্তিত্বের আদর্শ, মহত্ত্য, শৌর্য বীর্য প্রকাশিত হয়েছে।

‘কাব্য আমপারা’ নজরুল ইসলামের একটি উল্লেখযোগ্য রচনা। এটি পবিত্র কুরআন শরীফের আংশিক বাংলা অনুবাদ। ‘কাব্য-আমপারা’ সম্পর্কে নজরুল বলেছেন:

আমার জীবনে সবচেয়ে বড় সাধ ছিল পবিত্র ‘কুরআন’ শরীফের বাঙলা পদ্যানুবাদ করা। ... আমার বিশ্বাস, পবিত্র কুরআন শরীফ যদি সরল বাঙলা পদ্যে অনূদিত হয়, তাহলে তা অধিকাংশ মুসলমানই সহজে কঠ্ঠস্থ করতে পারবেন-অনেক বালক বালিকাও সমস্ত কুরআন হয়ত মুখস্ত করে ফেলবে। কাব্য আমপারা সম্পর্কে নজরুল গবেষক কামরুল আহসান বলেন, 
এ গ্রন্থে ‘খাদেমুল ইসলাম’ হিসেবে নিজের পরিচয় উল্লেখের ভেতর দিয়ে কবি তার পরিবর্তিত মানসিকতারই প্রতিফলন ঘটিয়েছেন এবং শানে নজুল সংযুক্ত আটত্রিশটি সুরার যাচাইকৃত সুললিত পদ্যানুবাদ করে আপন ইচ্ছার বাস্তবায়ন করেছেন। তবে মনে রাখা দরকার যে, এটি কবির কোনো মৌলিক গ্রন্থ নয়। মুসলমানদের পবিত্র ধর্ম গ্রন্থের আংশিক অনুবাদ মাত্র। এ কারণে নজরুলের কবিত্বগুণের প্রকাশ এতে থাকলেও মৌলিক ইসলামি দৃষ্টিভঙ্গির কোনো ছাপ না থাকাই স্বাভাবিক। (কামরুল আহসান, নানা ভাবনায় নজরুল)

উপর্যুক্ত দু’টি উদ্ধৃতির ভেতর ‘কাব্য আমপারার’ মর্মার্থ স্পষ্ট। এখানে একটি কথা বলা জরুরি, এই কাব্য আমপারাটি যদি নজরুল পূর্ণ করতে পারতেন তাহলে হয়ত মুসলিম বিশ্বে পবিত্র কুরআন শরীফের তাৎপর্য অত্যন্ত সহজবোধ্য হয়ে প্রতিটি বাংলা ভাষা-ভাষী মুসলমান মানুভের কাছে অবিস্মরণীয় ভূমিকা রাখতে পারতো।

নজরুলের অপর একটি কীর্ত ‘মরুভাস্কর’ কাব্য। হযরত মুহাম্মদ সা.-এর জীবনী নির্ভর করে লেখা হয়েছে এ কাব্যটি। এটি প্রথম গ্রন্থাকারে প্রকাশিত হয় ১৯৫০ সালে। মরুভাস্কর প্রসঙ্গে কবি স্ত্রী প্রমীলা বলেছেন:

অনেকদিন আগে দার্জিলিং এ বসে কবি এই কাব্য গ্রন্থখানি রচনা আরম্ভ করেন। তিনি তখন আধ্যাত্মিকভাবে নিমগ্ন। বিশ্বনবি হযরত মুহাম্মদ সা.-এর জীবনী নিয়ে একখানি বৃহৎ কাব্য গ্রন্থ রচনার কথা তিনি প্রায়ই বলতেন । ১৫

মরুভাস্কর কাব্যটি কাহিনী কাব্য বা আখ্যায়িকা ধর্মী মহাকাব্য। তবে যাই হউক না কেন কোনোটিই পূর্ণাঙ্গ নয়। কারণ, নজরুল একাটানা কাব্যটি রচনা করতে পারেননি। হযরত মুহাম্মদ সা.-এর বাল্য, কৈশোর ও যৌবনকালের পঁচিশ বছরের ইতিহাস রচনা করার পর নজরুলের লেখনি শক্তি থেমে যায়। নবিজির পঁচিশ বছরের ইতিহাস নজরুল চারটি সর্গের ভেতর আঠারটি পরিচ্ছেদে বর্ণনা করেছেন ।

“মরুভাস্কর” মহাকাব্যের আগিকে নজরুল রচনা করতে শুরু করেছিলেন। কিন্তু এটি অর্ধেক শেষ করার পরই নজরুলের লেখনি শক্তি হারিয়ে যায়। তারপরও বলা যায়, এখানে আমরা আমাদের মুসলিম জাহানের এবং ইসলানের যে ইতিহাস পাই তা নজরুল সাহিত্যের একটি গুরুত্বপূর্ণ অধ্যায়।

সমকালীন রাজনৈতিক অস্থিরতা, অর্থনৈতিক ও সমাজিক নানারকম অসঙ্গতি থেকে মুক্তি কামনায়ও নজরুলের বিদ্রোহী কবিকন্ঠ সোচ্চার। “জিঞ্জীর” কাজী নজরুল ইসলানের ইসলামি ঐতিহ্য বিষয়ক কবিতা ও গানের সংকলন। অগ্নিবীণা, বিষের-বাঁশি, ভাঙার গান কাব্যে ইসলামি ঐতিহ্য বিষয়ক কবিতা ও গানে নজরুল ইসলামের সং্রামী ও রাষ্ট্রীয় আদর্শকে জাগ্রত করে বর্তমানকে সমৃদ্ধ করার যে প্রয়াস দেখিয়েছেন জিজ্জীরের রচনায় সে প্রয়াস অধিকতর বেগবান। গ্রন্থের খালেদ, চিরঞ্জীব জগলুল, আমানুল্লাহ, উমর ফারুক ইত্যাদি কবিতায় ইসলামের অতীত ঐতিহ্যকে জাগ্রত করে বর্তমানকে অনুপ্রাণিত করে তোলার প্রচণ্ড প্রয়াসই লক্ষণীয়। ‘ঈদ-মোবারক’ কবিতাটিতে সাম্যের যে আহবান কবি করেছেন তা অসাধারণ। ইসলামের মহান আদর্শাবলীকে উজ্জীবিত করে এ কাব্যেও পরাধীনতা, সামপ্রদায়িতা, শ্রেণিভেদসহ নানা রকম অসামঞ্জস্যতা থেকে মুক্তির পথ দেখিয়েছেন ইসলানের বীর সেনানীদেরকে। 'আয় বেহেশ্তে কে যাবি আয়, ঈদ-মোবারক, আমানুল্লাহু, অগ্র-পথিক, বার্ষিক সওগাত, অঘ্রাণের সওগাত, নওরোজ, ভীরু ইত্যাদি জিঞ্জীর গ্রন্থের অপরাপর সংতোজন। যৌবন বন্দনা যৌবনের প্রতি জয়গান নজরুল মানসের একটি প্রধানতম বৈশিষ্ট্য। এ কাব্যের কবিতা ও গানে তেমনি যৌবনকে আলোকের 
পথে উদ্দীপিত করারও প্রয়াস রয়েছে। জিঞ্জীর সম্পর্কে স্পষ্টতই বলা যায়, এটি শুধু নজরুলের ইসলামি ঐতিহ্য বিষয়ক শ্রেষ্ঠ কাব্য নয়, এটি বাংলা কাব্যজগতেরও শ্রেষ্ঠ ইসলামি ঐতিহ্য বিষয়ক কাব্যগ্রন্থ।

\section{উপসংহার}

মুসলিম ঐতিহ্য ও নজরুল সাহিত্য শীর্ষক প্রবন্ধটিতে আলোচনা-পর্যালোচনায় দেখা যায়, নজরুল সাহিত্যে ইসলাম ধর্ম ও মুসলিম ঐতিহ্যের যে সমাহার রয়েছে তা বাংলা সাহিত্যে নজরুল পূর্ব এবং নজরুল পরবর্তী কারো সাহিত্যে খুঁজে পাওয়া যায় না। নজরংল কখনো ধর্মকে আবার কখনো ধর্মের নানা আনুসঙ্গিক বিষয়কে তার লেখনির বিষয়বস্ত হিসেবে নিয়েছেন। তিনি কখনো মুসলিম ঐতিহ্যেকে আবার কখনো কোনো মুসলিম আদর্শকে তার লেখনির বিষয়বস্তু হিসেবে নিয়েছেন। বাংলা সাহিত্যের প্রায় প্রতিটি শাখায় নজরুল নানাভাবে ইসলাম এবং ধর্মীয় অনুসঙ্গের মনোমুগ্ধকর জোয়ার বয়ে দিয়েছেন। তারুণ্যকে সঠিক পথের ঠিকানা দিয়েছেন নজরুল তার এই মুসলিম ঐতিহ্য বিষয়ক রচনার মধ্য দিয়ে।

\section{Endnotes}

1. Ahsan, K. (2001), Nana Vabonai Nazrul, Afsar Brothers, Bangal Bazar, Dhaka, p. 72.

2. Ibid.

3. Ibid, p. 74 .

4. Hossain, M. (2001). Nazrul-er Maru Bhaskar Kabbo, Nazrul Institute, p. 64.

5. Nazrul Rochona Samvar. (1997). Hajrat Prokashoni, p. 214.

6. Ibid, p. 227.

7. Ibid, p. 201.

8. Ibid, p. 229.

9. Ibid, p. 216.

10. Ibid, p. 227.

11. Ibid.

12. Ibid.

13. Chowdhury, A. M. (2001). Nazrul Islam: Islamic Kobita, Nazrul Institute, Pp. 5-6.

14. Islam, K. N. (1957). Kabbya Ampara (Collected).

15. Islam, K. N. (1957). Maru Bhaskar, Kolkata Edition (Noted from the Introductory Part).

16. Ahsan, K. (2001), Nana Vabonai Nazrul, Afsar Brothers, Bangal Bazar, Dhaka, p. 73. 